\title{
An assessment of the clinical acceptability of direct acoustic cochlear implantation for adults with advanced otosclerosis in the United Kingdom
}

Running head: Acceptability of DACI for Otosclerosis in the UK

Pádraig Thomas Kitterick PhD ${ }^{1,2}$, Guna Reddy-Kolanu BMBS $S^{3}$, David Baguley PhD', Jeremey Lavy FRCS $S^{4}$, Peter Monksfield FRCS ${ }^{5}$, Rupan Banga PhD ${ }^{5}$, Jaydip Ray PhD ${ }^{6}$, Ad Snik PhD ${ }^{7}$, Gerard M. O’ Donoghue FRCS $S^{1,2,8}$

${ }^{1}$ National Institute for Health Research Nottingham Hearing Biomedical Research Unit, Ropewalk House, Nottingham, United Kingdom

${ }^{2}$ Otology and Hearing group, Division of Clinical Neuroscience, School of Medicine, University of Nottingham, Nottingham, United Kingdom

${ }^{3}$ Royal United Hospitals Bath NHS Foundation Trust, Bath, United Kingdom

${ }^{4}$ Department of Otolaryngology, The Royal National Throat Nose and Ear Hospital, London, United Kingdom

${ }^{5}$ Department of Otolaryngology, University Hospitals Birmingham, United Kingdom

${ }^{6}$ Department of Otolaryngology, Royal Hallamshire Hospital, Sheffield Teaching Hospitals, Sheffield, United Kingdom

${ }^{7}$ Department of Otorhinolaryngology, Radboud University Medical Center, Nijmegen, The Netherlands

${ }^{8}$ Nottingham University Hospitals NHS Trust, Queen's Medical Centre, Nottingham, United Kingdom

Address correspondence to: Pádraig Kitterick

National Institute for Health Research Nottingham Hearing Biomedical Research Unit Ropewalk House, 113 The Ropewalk, Nottingham, NG1 5DU, United Kingdom Telephone: 00441158232626, Fax: 00441158232615

Email: padraig.kitterick@,nottingham.ac.uk

\section{Sources of Funding:}

This work was funded by a research grant from Cochlear Ltd. and infrastructure funding from the National Institute for Health Research (NIHR). The views expressed are those of the author(s) and not necessarily those of the NHS, the NIHR or the Department of Health. 


\section{Introduction}

2 Permanent conductive hearing loss can result from otosclerosis, a disease in which abnormal

3 bone growth may impede the movement of the stapes bone and impair cochlear function.

4 Examinations of temporal bones suggest that the disease presents bilaterally in approximately

$5 \quad 70-80 \%$ of cases (Hueb et al., 1991; Menger \& Tange, 2003). Temporal bone studies have

6 estimated a prevalence for otosclerosis of between $2.5 \%$ (Declau et al, 2001) and 8.3\%

7 (Altman et al, 1967). These estimates represent the combined sum of both symptomatic

8 (clinical otosclerosis) and asymptomatic (histologic otosclerosis) cases. The proportion of

9 these cases that correspond to clinical otosclerosis, where the disease actually interferes with

10 hearing function, has been estimated to be between 12\% (Altman et al, 1967) and 15\%

11 (Guild, 1944). These data therefore suggest that the prevalence of clinical otosclerosis in the

12 population lies between $0.3 \%$ and $1.2 \%$. It is estimated that sensorineural hearing loss also

13 arises in about $10 \%$ of clinical otosclerosis cases (Browning \& Gatehouse, 1992; Ramsay \&

14 Linthicum, 1994) and accounts for approximately 5-6\% of cases at large cochlear implant centres (Tange R., personal communication, 2016), which if accurate would correspond to a

16 prevalence for mixed losses arising from otosclerosis of $0.1 \%$ or lower.

18 Several treatment options are available for adults with a bilateral mixed hearing loss of a

19 mild, moderate, or severe degree. If the hearing loss is mild, a conventional acoustic hearing

20 aid can be sufficient to overcome the conductive and sensorineural components. A hearing

21 aid may also be beneficial for a moderate-to-severe loss provided the aid can overcome the

22 conductive component while still providing sufficient residual amplification to aid the

23 sensorineural component. The conductive component of the loss may also be addressed by

24 performing stapes surgery where a prosthesis is placed to restore the function of the fixed

25 stapes bone. The sensorineural component may then be more readily aided using an acoustic 
hearing aid. In cases where an acoustic hearing aid cannot provide sufficient amplification or is not tolerated (e.g. ear infections) or surgical correction is not appropriate, a bone-anchored hearing device (BAHD) may be used to deliver acoustical energy to the cochlea via bone conduction (Tjellström \& Håkansson, 1995). In cases of moderate-to-severe loss where both acoustic and bone-anchored hearing aids are unsuccessful or contraindicated, a middle-ear implant may also be considered.

In the United Kingdom (UK), the treatment options for individuals with a severe-to-profound mixed hearing loss are limited. The severity of the loss means that an acoustic hearing aid alone is unlikely to provide benefit without surgical intervention to address the conductive component. The rate of successful stapes surgery in this patient group has been estimated to be approximately $60 \%$ (defined as the closure of the air-bone gap to $<10 \mathrm{~dB}$ ) and lower than that observed in patients with mild or moderate losses (Kisilevsky et al, 2010). The capacity of a BAHD device to provide benefit in these patients is also limited by its ability to provide sufficient energy transfer to the cochlea to overcome the sensorineural component of the loss. The introduction of more powerful BAHDs has expanded the candidacy range but aiding those with more severe sensorineural losses is still restricted by feedback (Bosman et al, 2006). Although individuals with bone-conduction thresholds between 60-90 dB HL are therefore unlikely to be aided satisfactorily by either acoustic or bone-anchored hearing aids, they also do not meet current UK candidacy criteria for cochlear implantation (NICE, 2009).

The Direct Acoustic Cochlear Implant (DACI) was developed to address this gap in treatment options for individuals with a severe-to-profound mixed hearing loss (Häusler et al., 2008). The DACI is an active implantable device which is composed of two parts. The whollyimplanted part comprises a receiver-stimulator and a fixation system that couples an artificial 
incus to a conventional stapes prosthesis (Fig. 1). The external part comprises a speech processor that converts incoming sound into a digital signal that is transmitted to the implanted part via a radio-frequency coil. The receiver-stimulator decodes that digital signal and drives the actuator accordingly via a mechanical piston. By stimulating the intracochlear fluids directly, the DACI bypasses any existing conduction problems in the middle ear and can deliver acoustical energy directly to the cochlear perilymph of sufficient power to aid severe-to-profound sensorineural losses.

Lenarz et al. (2013) conducted a case series study of the safety and efficacy of the DACI in 15 patients with a severe-to-profound mixed hearing loss defined as bone-conduction thresholds poorer than $30 \mathrm{~dB}$ HL from 0.5 to $4 \mathrm{kHz}$ and an air-bone gap of at least $30 \mathrm{~dB}$ at 3 or more test frequencies. On average, implantation of the device did not impair air- or boneconduction thresholds, with bone-conduction thresholds improving at $0.75,1$, and $1.5 \mathrm{kHz}$ post-operatively. The DACI also improved sound-field thresholds measured from $250 \mathrm{~Hz}$ to 8 $\mathrm{kHz}$. Among those patients who used a hearing aid pre-operatively, the DACI improved sound-field thresholds, sentence recognition, and word recognition in quiet. The results of this preliminary study suggest that the DACI may be efficacious in patients with a severe-toprofound mixed hearing loss and with moderate bone-conduction thresholds (Busch et al., 2013).

Direct acoustic cochlear implantation is not currently provided in the UK. Evidence from a well-designed prospective evaluation of effectiveness that compared DACI to usual care would be required to support its provision. However, there is uncertainty over which comparator intervention(s) should be used to represent usual care. There is also uncertainty over the audiometric definition of the patient group whose needs are unmet by usual care and 
who would therefore be included in the future trial. Finally, there is uncertainty over whether clinicians in the UK would support such a trial. A study was therefore conducted to address these areas of uncertainty and to inform the design of the future trial.

\section{Materials and Methods}

An online survey was constructed using the Survey Monkey ${ }^{\mathrm{TM}}$ software. The patient group of interest was defined in accordance with the indications for the Codacs ${ }^{\mathrm{TM}} \mathrm{DACI}$ manufactured by Cochlear Ltd., Sydney, Australia (Cochlear 2013) as follows: (a) Otosclerosis; (b) Bone conduction (BC) thresholds of $55 \mathrm{~dB}$ or worse; (c) Air conduction thresholds in the severe-toprofound range; (d) Receive insufficient benefit from conventional hearing aids. It was also clarified that these patients should be assumed to be otherwise healthy and that they fall outside the candidacy guidelines for cochlear implantation in the UK following guidance from the National Institute for Health and Care Excellence (NICE 2009).

An initial question asked about the professional group to which respondents belonged (ENT, Audiologist, Hearing therapist, Other) as this survey sought to explore the routine practice and views of the various professional groups responsible for the care of these patients.

93 Respondents were then asked to consider a vignette that described the patient group of interest and indicate the preferred treatment option for these patients (Fig. 2). The treatment options were given as: 'No intervention', 'Audiological / speech-language therapy', 'Amplification with hearing aids', 'Combination of amplification and audiological / speech-

97 language therapy', 'Other (please specify)', and 'I don't know'. Respondents to the survey were also asked to indicate the important outcomes to assess when measuring clinical benefit in the patient group of interest. The available outcome domains were specified based on a 
100 review of those assessed in previous clinical studies of DACI (Busch et al 2013; Lenarz et al 1012013 ) and are listed in Table 1.

103 Finally, respondents were reminded of the characteristics of the patient group of interest

104 before being asked three questions about the clinical appropriateness of the DACI device, 105 whether respondents would be willing to refer these patients into a trial of the DACI device, 106 and at what stage in their treatment would they be willing to refer. For the latter, the options

107 given were: 'Even before initial stapes surgery', 'Only after stapes surgery’, 'Only after 108 revision stapes surgery', 'Other (please specify)', and 'I don't know'. The DACI device was 109 not described by name but rather as a device which: (a) Couples directly to the perilymph of

110 the cochlea via a conventional stapes prosthesis; (b) Is capable of delivering sufficient gain to

111 aid bone conduction thresholds of $55 \mathrm{~dB}$ or worse; (c) Involves the surgical placement of a

112 receiver/stimulator similar to that of a cochlear implant; and (d) Involves the use of a behind-

113 the-ear sound processor similar to that used with a cochlear implant.

115 A consensus process was conducted to identify inclusion criteria for a future trial of direct 116 acoustic cochlear implantation. An initial face-to-face meeting of experts in otosclerosis was

117 held at which attendees were presented with information on the surgical considerations and

118 audiological management by clinical professionals who have experience with providing

119 DACI. A facilitated discussion was then held around three topics: 'Which patients do not

120 benefit from current treatment options in the UK?', 'Who are potential candidates for

121 DACI?', and 'What factors should guide the design of a future trial and would it be feasible?'

122 A transcript of the resulting discussions was analysed and used to generate statements around

123 which a potential consensus could be reached. Two rounds of an online survey were

124 conducted. In the first round, respondents were asked to state their level of agreement with 
125 each of the resulting statements on a five-point Likert scale from 'Strongly disagree' to

126 'Strongly agree'. In the second round, respondents were shown the level of agreement that

127 had been expressed in round 1 and asked to reconsider their response in light of that

128 information. Consensus was considered to have been reached on a particular statement if at

129 least $80 \%$ of respondents agreed with it.

130

131 The survey and consensus exercise were advertised through national professional bodies:

132 ENT UK for otolaryngologists, and both the British Academy of Audiology and British

133 Society of Audiology for audiologists. Invitations to participate were also sent directly to

134 clinicians working at major referral centres for otosclerosis in the UK.

136 Results

137 Thirty-two clinical professionals completed the online survey comprising nine ENT

138 specialists, 22 audiologists, and one hearing therapist. All had experience of managing

139 patients with advanced otosclerosis within the UK National Health Service (NHS). Of those,

14030 provided responses to the question about the preferred management options for patients

141 with advanced otosclerosis (Fig. 2). All but two (93\%; 95\% CI 78.7 to 98.2) indicated that

142 their preferred management would include amplification via conventional acoustic hearing

143 aids with $11(37 \% ; 95 \% 21.9$ to 54.5$)$ also indicating that they would recommend hearing

144 therapy in addition to amplification. Only two respondents suggested alternative treatment

145 options, which were the provision of a bone anchored hearing device and cochlear

146 implantation.

148 Respondents' choices for the most important outcome to assess when measuring treatment

149 benefit are shown in Table 1 . No outcome domain was chosen by a statistical majority of 
150 respondents either as the most or second most important outcome. The most frequently

151 chosen outcome across either response option was self-reported quality of life, with $55 \%$ of

152 respondents (95\% CI 37.5 to 71.6$)$ selecting it as either the most or second most important

153 outcome to assess treatment benefit.

154

155 When asked about whether DACI would be an appropriate treatment option for the patient group of interest, 25 of the 29 respondents $(86 \%$; 95\% 69.4 to 94.5$)$ indicated that it was, with the remainder selecting 'I do not know'. None indicated that it was inappropriate. All those who considered it appropriate also indicated a willingness to refer their patients into a

159 future trial. However, there was variability in when respondents would be willing to refer 160 patients with $9(38 \%$; $95 \% 21.2$ to 57.3$)$ willing to do so even before stapes surgery had been attempted and $8(33 \% ; 95 \% 18.0$ to 53.3$)$ willing only after stapes surgery had been carried out. One respondent indicated that they might be willing to refer before stapes surgery but only if further evidence for the effectiveness of the DACI was available. Three respondents

164 listed other criteria for referral, which were: (1) only after revision stapes surgery; (2) only after discussion with the patient; and (3) only after full investigation of non-surgical aiding options.

Nineteen clinical professionals participated in the consensus exercise. An analysis of the transcript of the face-to-face facilitated discussion identified sixteen statements around which consensus was considered possible. Table 2 lists these statements along with the levels of agreement after one and two rounds of voting. The consensus was that stapes surgery, either with or without a hearing aid, is the best available treatment for advanced otosclerosis and a hearing aid trial is recommended prior to surgery, if that patient is willing. Bone-anchored

174 hearing devices are an option for some patients and a headband trial would always 
175 recommended, but the limit of candidacy for these devices is considered to be BC thresholds at $50 \mathrm{~dB}$ HL. Bone-anchored hearing devices are considered to be not powerful enough for patients whose $\mathrm{BC}$ thresholds are greater than $55 \mathrm{~dB}$ HL.

179 The consensus was that there is a lack of clear alternative treatment options for those who

180 have already received the best available treatment, who are outside criteria for both bone conduction hearing devices and cochlear implantation, and who still receive insufficient benefit from their hearing aids. These patients would therefore be referred for an implantable intervention such as a DACI as long as the odds of the patient receiving additional benefit over their hearing aids were favourable and similar to those expected for benefit from a cochlear implant. The consensus was also that further trials are needed and that would be supported by clinical professionals involved in the management of these patients.

\section{Discussion}

189 It is perhaps as informative to examine the statements that did not reach the required level of

190 agreement as it is to identify where consensus was reached. The survey responses suggest

191 that stapes surgery would still be offered to some patients with an air-bone gap as small as 20

$192 \mathrm{~dB}$. The willingness of respondents to carry out stapes surgery even when benefit could be limited due to poor cochlear function could reflect the fact that pre-operative bone conduction levels may under-estimate the actual benefit achievable from stapes surgery (Shea et al., 1999). However, the observed consensus on the need for favourable odds of improvement to

196 warrant referral for a DACI suggests that there will be a lower limit of cochlear function

197 beyond which clinicians will not be willing to refer patients. It is therefore important for 198 future studies to characterise the relationship between pre-operative speech perception and 199 the odds of a favourable outcome following the provision of a DACI device. Such an 
approach can be used to define candidacy criteria based on the likelihood that the patient will improve following the intervention (UKCISG, 2004). However, studies should also consider the size of change that would be considered meaningful from clinical and patient perspectives.

It would also seem logical to assume that there will be an upper limit for the speech perception abilities of these patients beyond which DACI would either been seen as unnecessary or inappropriate. However, consensus was not reached on a statement that restricted referral to those with speech discrimination up to $50 \%$ correct, a threshold that has previously been used to define insufficient benefit from acoustic hearing aids in patients with

210 more profound losses (NICE 2009). The failure to reach consensus on this point could reflect

211 a belief that the threshold for referral should be more or less restrictive, but it could also be

212 that respondents believed 'insufficient benefit from hearing aids' cannot be defined

213 adequately or reliably in terms of a fixed threshold on a test of speech perception conducted

214 in the artificial environment of an audiology testing booth. In the absence of an agreed

215 threshold, such a judgement could be based on patient self-report of benefit in real life

216 situations following the confirmed completion of a hearing aid trial.

218 The failure to reach a consensus on whether clinicians were willing to refer for a DACI where

219 a conductive component remained suggests that referral would be conditional on the outcome 220 of stapes surgery in those patients where surgery would be recommended. However, the group failed to reach consensus on a general statement indicating that stapes surgery would be required before referral for a DACI could be recommended. This result is compatible with the fact that the needs of patients for whom stapes surgery is not recommended were

224 considered to be unmet by the available treatment options. Their apparent willingness to refer 
some patients for a DACI even without having conducted stapes surgery could also have reflected their views on the needs of patients with losses that are predominantly sensorineural in origin. The current study did not ask about such patients as it fell outside the current labelling of the device at the time the study was conducted (Cochlear, 2013).

230 The current study aimed to inform the design of a future trial of DACI in the UK, including identifying the target patient population for whom the intervention is appropriate and needed. Table 3 lists proposed inclusion and exclusion criteria for a trial based on an analysis of the statements upon which the respondents reached consensus. The results of the current practice survey and the consensus exercise both suggest that the trial design needs to account for two groups: (1) those for whom stapes surgery is recommended where referral for a DACI would only be supported after that surgery has been conducted; (2) those for whom stapes surgery would not be clinically appropriate and for whom referral would be supported without prior surgical intervention. In both cases, the comparator to the DACI should be a trial of an acoustic hearing aid in combination with hearing therapy. Respondents' views on important outcome domains suggest that the primary end-point for the trial should be an assessment of quality of life. Previous early-phase evaluations of the DACI have used a well-established measure of the impact of listening difficulties on everyday life (the Abbreviated Profile of Hearing Aid Benefit (APHAB); Cox \& Alexander, 1995) and have suggest that outcomes

244 could be assessed as early as three months after the intervention is provided (Lenarz et al., 245 2013). The resulting trial design is shown in Figure 3.

247 Prior to conducting such a trial, a feasibility study would be required to assess such practical 248 issues as the learning curves of surgeons, the structure of the clinical pathway following 249 provision of a DACI, and the nature and content of post-operative rehabilitation that would 
be required. The willingness of patients to accept both randomization and the intervention itself would also need to be confirmed. Should a randomized controlled trial be unacceptable to patients or not be feasible to conduct, alternative approaches such as the creation of a matched control group from existing patients populations using propensity score matching could be considered (McCulloch et al., 2009). In that approach, patients are drawn from a control group based on their similarity to a smaller group of patients who receive the treatment on factors that could influence outcome.

The current study identified quality of life as the outcome domain most frequently chosen by respondents. This result is one of two key pieces of information that are necessary to

260 determine the required sample size for the future trial (Williamson et al 2012). The other is the smallest difference on that outcome that could be considered to be clinically important and is referred to as the minimal clinically-important difference (MCID) (Gatchel et al 2010). While the most important outcome domain can be identified through the use of surveys and consensus techniques (Sinha et al 2011), as demonstrated in the current study, the MCID is determined by relating the change in outcome to whether the patient perceived a change or not. The size of the change in outcome among those reporting no change in their hearing provides an estimate of the minimally-important difference (Jaeschke et al 1989). Further work would be required to identify an instrument that measures those aspects of quality of life that are relevant to the specific patients of interest (Buchbinder et al., 2011). Early-phase studies have already suggested that the APHAB is sensitive to the reductions in everyday listening difficulty that occur following the provision of a DACI (Lenarz et al., 2013).

273 The current study suggests that there is a patient population for whom there is a lack of 274 treatment options and for whom direct stimulation of the cochlea via the implantation of an 
275 auditory prosthesis is considered an appropriate intervention. There appears to be strong

276 support amongst the clinical professionals who manage the care of these patients to conduct a

277 clinical trial to evaluate the effectiveness of this novel intervention. A feasibility study is now

278 necessary to determine how many patients would be required for that future trial, whether

279 those patients could be recruited within a reasonable timeframe, and whether the proposed

280 trial design would be acceptable to patients.

281

282 


\section{Acknowledgements}

284 The authors wish to thank Cochlear Europe Ltd. for the financial resources to conduct this 285 feasibility study and the support of Lisa DeBold and Lisa Aubert. The authors also wish to 286 acknowledge the valuable contributions made to the study by Martin O'Driscoll and Kevin

287 Green (Central Manchester University Hospitals), Joanne Muff (Cambridge University 288 Hospitals), Chris Aldren (BMI The Princess Margaret Hospital), John Oates (Spire Little 289 Aston Hospital), Steve Broomfield (University Hospitals Bristol), Terry Nunn (Guy’s and St.

290 Thomas' Foundation Trust), and Shakeel Saeed (University College London Hospitals).

\section{References}

293 Altmann F, Glasgold A, Mcduff JP. The incidence of otosclerosis as related to race and sex. Ann Otol Rhinol Laryngol 1967;76:377-92.

Bosman AJ, Snik AF, Mylanus EA, Cremers CW. Fitting range of the BAHA Cordelle. Int J Audiol 2006;45(8):429-37.

Browning GG, Gatehouse S. The prevalence of middle ear disease in the adult British population. Clin Otolaryngol Allied Sci 1992;17(4):317-21.

Browning GG, Gatehouse S. Sensineural hearing loss in stapedial otosclerosis. Ann Otol Rhinol Laryngol 1984;93:13-16.

Buchbinder R, Batterham R, Elsworth G, et al. A validity-driven approach to the understanding of the personal and societal burden of low back pain: development of a conceptual and measurement model. Arthritis Res Ther 2011;13(5):1.

304 Busch S, Kruck S, Spickers D, et al. First clinical experiences with a direct acoustic cochlear stimulator in comparison to preoperative fitted conventional hearing aids. Otol Neurotol 2013;34(9):1711-8. 
307 Cochlear. Surgical Instructions For Use: Codacs ${ }^{\mathrm{TM}}$ DI1 10 Implant system. Cochlear Limited, $308 \quad$ New South Wales, Australia, 2013.

309 Cox RM, Alexander GC. The abbreviated profile of hearing aid benefit. Ear Hear $310 \quad 1995 ; 16: 176-186$.

311 Declau F, Van Spaendonck M, Timmermans JP, et al. Prevalence of otosclerosis in an 312 unselected series of temporal bones. Otol neurotol 2001;22(5)596-602.

313 Gatchel RJ, Lurie JD, Mayer TG. Minimal clinically important difference. Spine 2010;35(19):1739-1743.

315 Guild SR. Histologic otosclerosis. Ann Otol Rhinol Laryngol 1944;53:246-67.

316 Häusler R, Stieger C, Bernhard H, et al. A novel implantable hearing system with direct 317 acoustic cochlear stimulation. Audiol Neurootol 2008;13(4):247-56.

318 Hueb MM, Goycoolea MV, Paparella MM, et al. Otosclerosis: The University of Minnesota 319 temporal bone collection. Otolaryngol Head Neck Surg 1991;105(3):396-405.

320 Jaeschke R, Singer J, Guyatt GH. Measurement of health status: ascertaining the minimal clinically important difference. Control clin trials 1989;10(4):407-415.

Kisilevsky VE, Bailie NA, Halik JJ. Results of stapedotomy in otosclerosis with severe and profound hearing loss. J Otolaryngol Head Neck Surg 2010;39(3):244-52.

Lenarz T, Zwartenkot JW, Stieger C, et al. Multicenter study with a direct acoustic cochlear implant. Otol Neurotol 2013;34(7):1215-25.

McCulloch P, Altman DG, Campbell WB, et al. No surgical innovation without evaluation: the IDEAL recommendations. Lancet 2009;374(9695):1105-1112.

Menger DJ, Tange RA. The aetiology of otosclerosis: a review of the literature. Clin Otolaryngol Allied Sci 2003;28(2):112-20.

330 National Institute for Health and Care Excellence (NICE). Technology appraisal guidance 166: Cochlear implants for children and adults with severe to profound deafness. 2009. 
332 Ramsay HAW, Linthicum FH. Mixed hearing loss in otosclerosis: indication for long-term follow-up. Am J Otol 1994; 15:536-539.

334 Shea PF, Ge X, Shea Jr JJ. Stapedectomy for far-advanced otosclerosis. Otol Neurotol $1999 ; 20(4): 425-429$.

336 Sinha IP, Smyth RL, Williamson PR. Using the Delphi Technique to Determine Which Outcomes to Measure in Clinical Trials: Recommendations for the Future Based on a Systematic Review of Existing Studies. PLoS Med 2011;8(1):e1000393.

Somers T, Govaerts P, Janssens de Varebeke S, et al. Revision stapes surgery. J Laryngol Otol 1997;111:223-39.

341 Tjellström A, Håkansson B. The bone-anchored hearing aid. Design principles, indications, and long-term clinical results. Otolaryngol Clin North Am 1995;28(1):53-72.

343 Williamson PR, Altman DG, Blazeby JM, et al. Developing core outcome sets for clinical trials: issues to consider. Trials 2012;13(1):132. 


\section{Figure captions}

346 Figure 1: A photograph of the implanted component of a direct acoustic cochlear

347 implant system (left) and a computer rendering of the fixation system (right) used to

348 attach the mechanical actuator (5) to a conventional stapes prosthesis. 1: Removable

349 magnet; 2: Receiver coil; 3: implant electronics; 4: lead assembly; 5: actuator; 6: rod; 7:

350 artificial incus. Reproduced from the surgical instructions for use (Cochlear, 2013).

351 Figure 2: The clinical vignette used to assess the preferred management option for the 352 target patient group with advanced otosclerosis. 

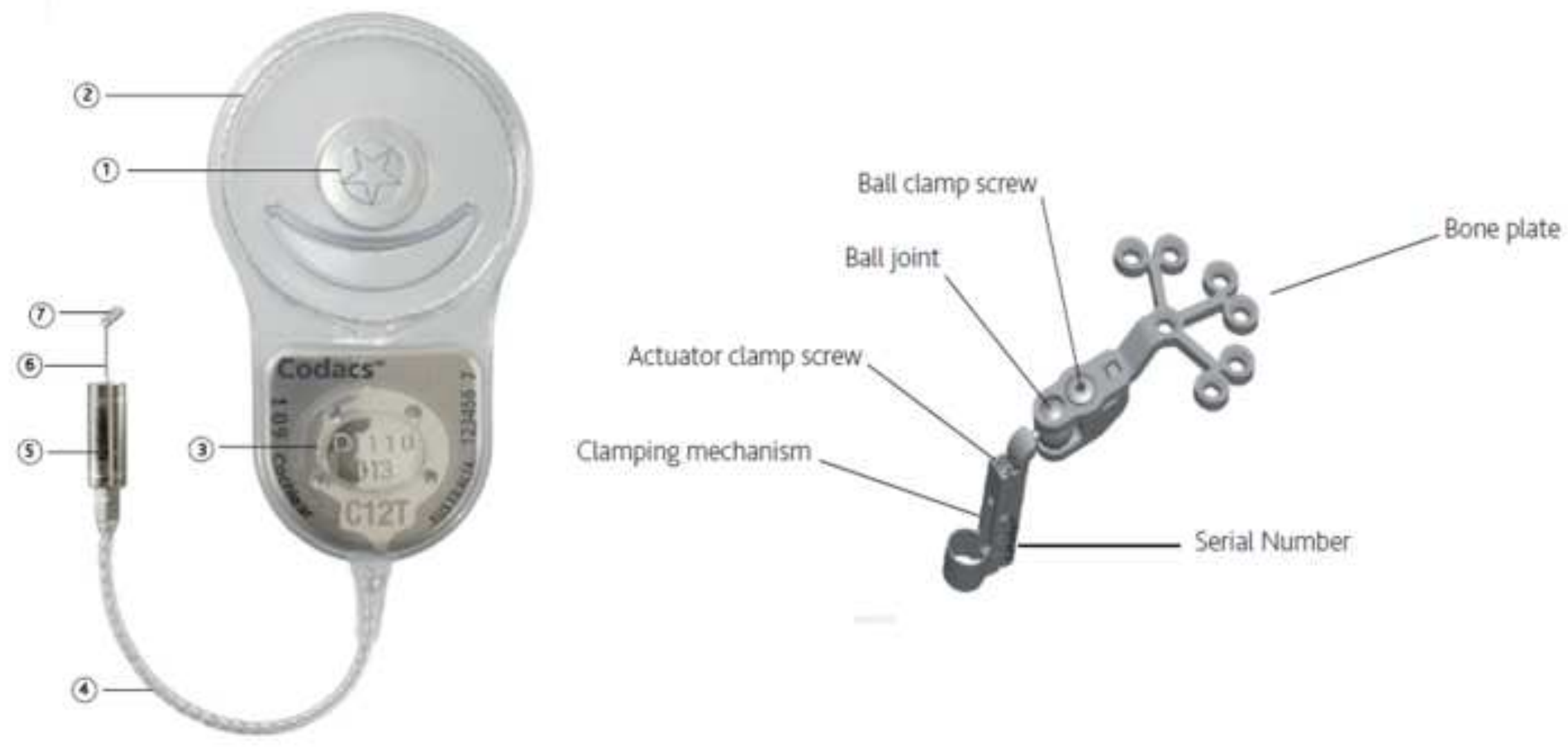
An otherwise healthy patient with otosclerosis currently wears two hearing aids. They have had stapes surgery which closed the air-bone gap to less than $10 \mathrm{~dB}$. Their post-operative audiogram is shown on the right. However, they still report receiving insufficient benefit from their hearing aids.

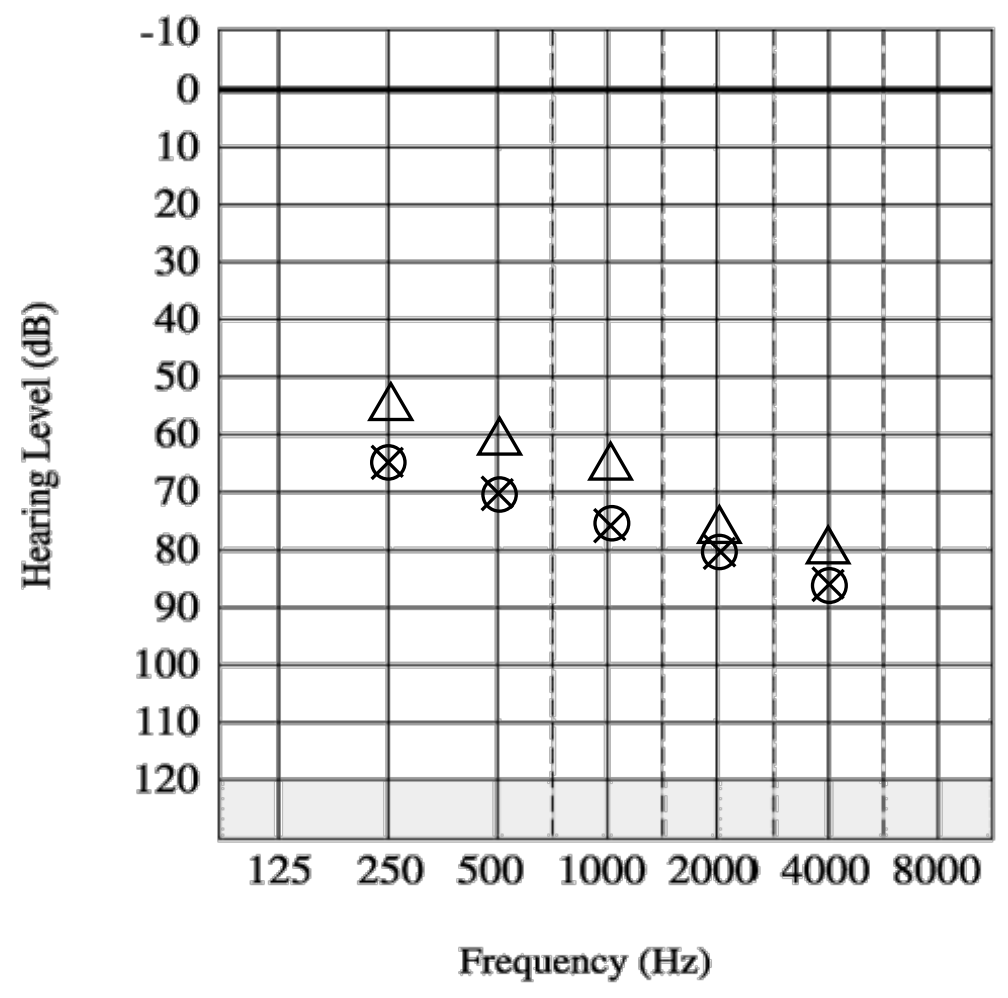




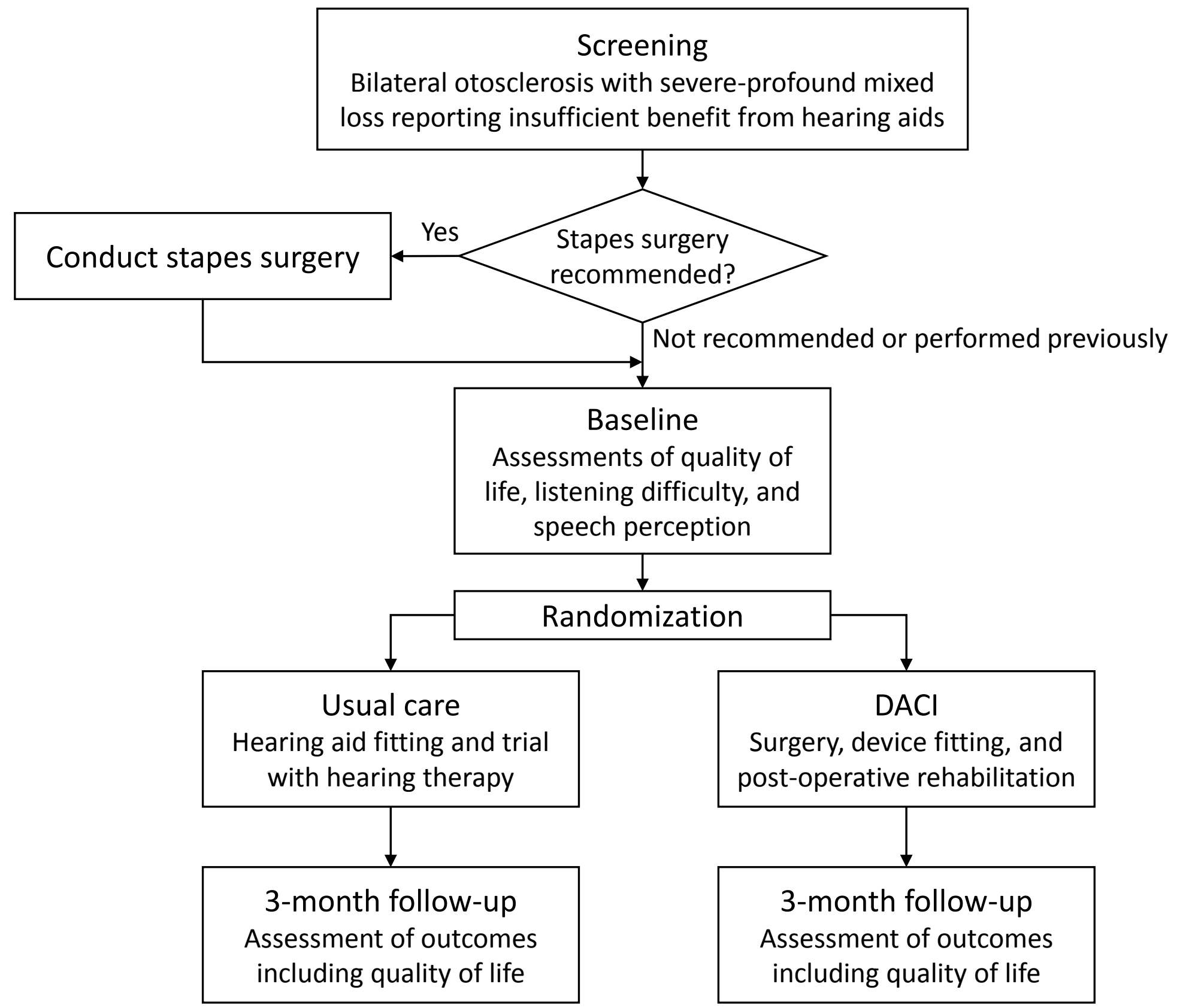


Table 1: Respondents choices for the most important outcome to assess when measuring treatment benefit in adults with advanced otosclerosis. The outcome domains have been sorted based on the proportion of respondents who identified them as the 'most important' outcome to measure to assess treatment effect. The values in parentheses represent the number of respondents.

\begin{tabular}{lccc}
\hline Outcome domain & Most important & 2nd most important & Total \\
\hline Quality of life reported by the patient & $38 \%(11)$ & $17 \%(5)$ & $55 \%(16)$ \\
\hline Ability to understand speech in quiet listening conditions & $17 \%(5)$ & $21 \%(6)$ & $38 \%(11)$ \\
\hline Ability to understand speech in noisy listening conditions & $17 \%(5)$ & $14 \%(4)$ & $31 \%(9)$ \\
\hline Level of listening difficulty reported by the patient & $14 \%(4)$ & $17 \%(5)$ & $31 \%(9)$ \\
\hline I don't know & $7 \%(2)$ & $0 \%(0)$ & $7 \%(2)$ \\
\hline Ability to localise sounds (tell where they are coming from) & $3 \%(1)$ & $0 \%(0)$ & $3 \%(1)$ \\
\hline Level of effort required to listen reported by the patient & $3 \%(1)$ & $10 \%(3)$ & $14 \%(4)$ \\
\hline Sensitivity to sound (e.g. pure-tone/soundfield audiometry) & $0 \%(0)$ & $7 \%(2)$ & $7 \%(2)$ \\
\hline Other & $0 \%(0)$ & $0 \%(0)$ & $0 \%(0)$ \\
\hline
\end{tabular}


Table 2: Level of agreement across 19 participants in the consensus process with 16 statements generated from the initial open round.

\begin{tabular}{|c|c|c|}
\hline Statement & $\begin{array}{l}\text { Round } \\
1\end{array}$ & $\underset{2}{\text { Round }}$ \\
\hline I would always recommend a hearing aid trial to patients with advanced otosclerosis before stapes surgery, as long as the patient is willing. & $93 \%$ & $95 \%$ \\
\hline $\begin{array}{l}\text { For otosclerosis patients with } \mathrm{BC} \text { thresholds worse than } 55 \mathrm{~dB} \text { but who are also outside of } \mathrm{CI} \text { criteria, either a hearing aid alone or in combination } \\
\text { with stapes surgery is the best treatment that is currently available. }\end{array}$ & $93 \%$ & $95 \%$ \\
\hline $\begin{array}{l}\text { I would not recommend stapes surgery for cases of advanced otosclerosis with sloping high-frequency loss because the risks would outweigh the } \\
\text { potential benefits to speech perception. }\end{array}$ & $36 \%$ & $16 \%$ \\
\hline I would not recommend stapes surgery to patients with advanced otosclerosis if their speech discrimination is worse than $30 \%$ correct. & $57 \%$ & $37 \%$ \\
\hline $\begin{array}{l}\text { There is currently a lack of treatment options for otosclerosis patients with } \mathrm{BC} \text { thresholds worse than } 55 \mathrm{~dB} \text {, who are outside of } \mathrm{CI} \text { criteria, and } \\
\text { who still struggle with HAs after receiving stapes surgery or if surgery is not recommended. }\end{array}$ & $79 \%$ & $89 \%$ \\
\hline $\begin{array}{l}\text { For patients whose } \mathrm{BC} \text { thresholds are worse than } 55 \mathrm{~dB} \text { and who are not close to CI criteria, I would not recommend stapes surgery if their air- } \\
\text { bone gap is less than } 20 \mathrm{~dB} \text {. }\end{array}$ & $64 \%$ & $63 \%$ \\
\hline I would consider a bone-anchored hearing device for a patient with otosclerosis if their BC thresholds are better than $55 \mathrm{~dB}$. & $71 \%$ & $74 \%$ \\
\hline Patients with otosclerosis whose $\mathrm{BC}$ thresholds are $50 \mathrm{~dB}$ are approaching the limits of what a bone-anchored hearing device can aid. & $86 \%$ & $89 \%$ \\
\hline I would always recommend a headband trial before surgery to provide a bone-anchored hearing device. & $93 \%$ & $89 \%$ \\
\hline The acoustic gain of a bone-anchored hearing device is insufficient for otosclerosis patients with BC thresholds worse than $55 \mathrm{~dB}$. & $86 \%$ & $89 \%$ \\
\hline I would always recommend stapes surgery to patients with advanced otosclerosis before referring them for a new implantable intervention. & $64 \%$ & $68 \%$ \\
\hline $\begin{array}{l}\text { I would not refer otosclerosis patients whose needs are currently unmet by currently-available treatments for a new implantable intervention if } \\
\text { their speech discrimination is better than } 50 \% \text { correct. }\end{array}$ & $57 \%$ & $26 \%$ \\
\hline $\begin{array}{l}\text { I would refer otosclerosis patients whose needs are currently unmet by currently-available treatments for a new implantable intervention, as long } \\
\text { as there is at least an } 80 \% \text { chance of the patient receiving additional benefit. }\end{array}$ & $71 \%$ & 89\% \\
\hline $\begin{array}{l}\text { I would refer otosclerosis patients whose needs are currently unmet by currently-available treatments for a new implantable intervention even if a } \\
\text { conductive component remained, as long as I am sure that their previous stapes surgery was done competently. }\end{array}$ & $64 \%$ & $68 \%$ \\
\hline Clinical trials are needed to evaluate new treatments for otosclerosis patients whose needs are currently unmet by currently-available treatments. & $100 \%$ & $100 \%$ \\
\hline I would support clinical trials to evaluate treatments for otosclerosis patients whose needs are currently unmet by currently-available treatments. & $100 \%$ & $100 \%$ \\
\hline
\end{tabular}


Table 3: Suggested inclusion and exclusion criteria for a trial of DACI in the United Kingdom.

\section{Inclusion criteria}

Bilateral severe-to-profound hearing loss defined as average $\mathrm{AC}$ thresholds $>70 \mathrm{~dB} \mathrm{HL}^{1}$

Bilateral otosclerosis

$\mathrm{BC}$ thresholds worse than $55 \mathrm{~dB} \mathrm{HL}$

Where recommended, has undergone stapes surgery that closed the air-bone gap to within $10 \mathrm{~dB}^{2}$

Completed a hearing aid trial

\section{Exclusion criteria}

Reports receiving sufficient benefit from acoustic hearing aids

Simultaneously satisfies both of the following criteria: ${ }^{3}$

1. A score of less than $50 \%$ on Bamford-Kowal-Bench (BKB) sentence testing at a sound intensity of $70 \mathrm{~dB}$ SPL

2. AC thresholds $>90 \mathrm{~dB}$ HL at 2 and $4 \mathrm{kHz}$

${ }^{1}$ Following definition of categories of hearing loss from British Society of Audiology (2011)

${ }^{2}$ Following definition of a resolved conductive component from Kisilevsky et al (2010)

${ }^{3}$ Following guidance on the candidacy criteria for cochlear implantation from NICE (2009) 